Proceedings

\title{
A Novel Low-Cost Method for Fabrication of 2D Multi-Electrode Array (MEA) to Evaluate Functionality of Neuronal Cells ${ }^{\dagger}$
}

\author{
Tala Ahmadvand 1, Sara Mirsadeghi 2,3, Faezeh Shanesazzadeh 1, Sahar Kiani ${ }^{2,3}$ and \\ Mehdi Fardmanesh 1,* \\ 1 Department of Electrical Engineering, Sharif University of Technology, Tehran, Iran; email1@gmail.com \\ (T.A.); email2@gmail.com (F.S.) \\ 2 Department of Stem Cell and Developmental Biology, Cell Science Research Center, Royan Institute for \\ Stem Cell Biology and Technology, ACECR, Tehran, Iran; email3@gmail.com (S.M.); email4@gmail.com \\ (S.K.) \\ 3 Department of Brain and Cognitive Science, Cell Science Research Center, Royan Institute for Stem Cell \\ Biology and Technology, ACECR, Tehran, Iran \\ * Correspondence: Fardmanesh@sharif.edu \\ + Presented at the 1st International Electronic Conference on Biosensors, 2-17 November 2020; Available \\ online: https://iecb2020.sciforum.net/.
}

Received: date; Accepted: date; Published: date

\begin{abstract}
In this paper, a fabrication method for two-dimensional multi-electrode arrays (MEAs) using inexpensive material and method is proposed. The focus in this work is on the design and fabrication of 2D Microelectrode arrays (MEAs) using metallic electrodes on a silica substrate. Titanium/Gold multi-electrode arrays containing 60 electrodes with optimized metal thicknesses and $30 \mu \mathrm{m}$ diameter, covered with thin modified SU-8 insulator layer as biocompatible material have been designed and manufactured using the standard photolithography-based microfabrication method. The utilization of affordable and more accessible materials and simpler techniques can be mentioned as the distinct point of the proposed method. Using these multielectrode arrays, it is possible to either record or stimulate cells by accessing multiple sites of cell tissues and collect signals from the sources around each electrode simultaneously. Precisely adjusting the size, distance, and the number of microelectrodes causes the high measurement selectivity and reliability which has been taken into account in the design of the microelectrodes. In this study, by implementing the proposed system, we manufactured a preliminary representative MEA and the bio-compatibility of the manufactured MEA is going to be evaluated by neural cells, obtained from rat cortices. The main aim of this study is comparison of our inexpensive strategy with other approaches.
\end{abstract}

Keywords: multi-electrode array; extracellular biosensor; biocompatibility; neural cell; extracellular recording

\section{Introduction}

Understanding the neuronal ensemble as well as their connectivity through recondite synapses in the brain or even in a limited regain of the nervous system is one of the most challenging topics in research over several past decades. In this regard, due to the development of microstructure technologies in the last 30 years, significant advances have been observed in tools and methods to measure the electrical activities of excitable cells (neurons and cardio-myocytes) both in vitro and in 
vivo, which gives the necessary knowledge to understand the characteristics and function of the excitable cells of the brain and heart.

Excitable cells generate the action potential by ion exchange through their membrane and multielectrode arrays are able to record the consequent fluctuations in the electrical field of the extracellular milieu of the cells. This non-invasive communication between the electrode and cell enables us to perform stimulation in addition to long-term recordings from the network. To date, the in vitro study of neural network electrical activity under physiological or pathological conditions largely relies on multi-electrode arrays technology. Due to the uniform distribution of the arrays and their non-invasive properties, this technology allows long-term studies at the neural network level to overcome the problems of conventional extrinsic electrophysiological techniques such as patch clamps. Indeed, the technology allows a precise understanding of the excitable network, drug screening, and neural prosthesis studies [1,2].

For electrical excitation and recording, electrodes with high selectivity, low impedance, high density, and multidimensional geometry are preferred to stimulate neurons and to achieve a high signal-to-noise ratio (SNR). During recording and stimulation, the impedance of the electrodes serves as the bridge of the current transducer, and less power and less background noise are resulted from the electrodes with lower impedance. This is while to achieve high selective small electrodes, the impedance should higher and the signal-to-noise ratio is lower. To solve these problems, we must consider a combination of all the main aspects, including the design of the geometry, the material, and the manufacturing techniques $[2,3]$.

Multi-electrode array was fabricated by two steps: mini MEA fabrication, and printed circuit board (PCB) with CMOS technology as the most expensive one [4,5]. Both methods involve complicated processes that lead to high-cost electrodes. For most laboratories conducting multiple studies, both commercially available and fabricated MEAs are too costly [3]. In this work, the plane microelectrodes were fabricated with conventional microfabrication processes including photolithography, metal sputtering, deposition of insulators, and etching. We are focusing on fabricating low-cost multi-electrode array chips using feasible material and microfabrication methods that are cost-effective for several laboratories. In the following sections, the design and fabrication process of low-cost high-density MEA are described. That is followed by the presentation of good cell adhesion and bio-compatibility of the manufactured MEA which is going to be evaluated by neural cells, obtained from rat cortices.

\section{Design of the Planar Microelectrodes Array}

The number of electrodes intended in multi-electrode arrays is the first parameter to be considered in its design which can be varied between 16 to 512 [6]. The greater number of electrodes may lead to a much more accurate recording plus more targeted manipulation of each individual cell in the network. In fact, by increasing the electrode figures, most regions of a single neuron comprising the dendrite, soma, and axon are accessible for either recording or stimulating which represent a perfect map of the neuronal ensemble as a result. Besides, more simultaneous recordings reduce the noise level of the system due to the differential relationship of the electrodes with each other. Additionally, whereas improving the quality of the signals requires enlarged dimensions of the electrodes, but this reduces the resolution of the received signals in terms of the position of the cells and their components. Therefore, the size of the electrodes in such a way to achieve the desired selectivity is another criterion in MEA design. In recent years, depending on the type of application, the size of the diameter of the electrodes is usually considered to be 10 to 50 microns $[4,6,7]$.

Subsequently, in this work, the number of electrodes is considered as 60 , on a square-shaped glass substrate with a side length of $5 \mathrm{~cm}$ and $1 \mathrm{~mm}$ thickness. As a standard size for the MEA chips, the diameter of electrodes is considered $30 \mathrm{~mm}$ and the contact pads are square with a side length of $200 \mathrm{~mm}$. On this substrate, we place a glass ring with an outer diameter of $2.4 \mathrm{~cm}$, an inner diameter of $1.9 \mathrm{~cm}$, and height of $6 \mathrm{~mm}$ to culture the cells within. 
The proposed pattern of 60 electrodes for planar multi-electrode array was designed using Advanced design system (ADS) and Layout editor softwares, shown in Figure 1. Then, we create a chrome mask for our photolithography process.

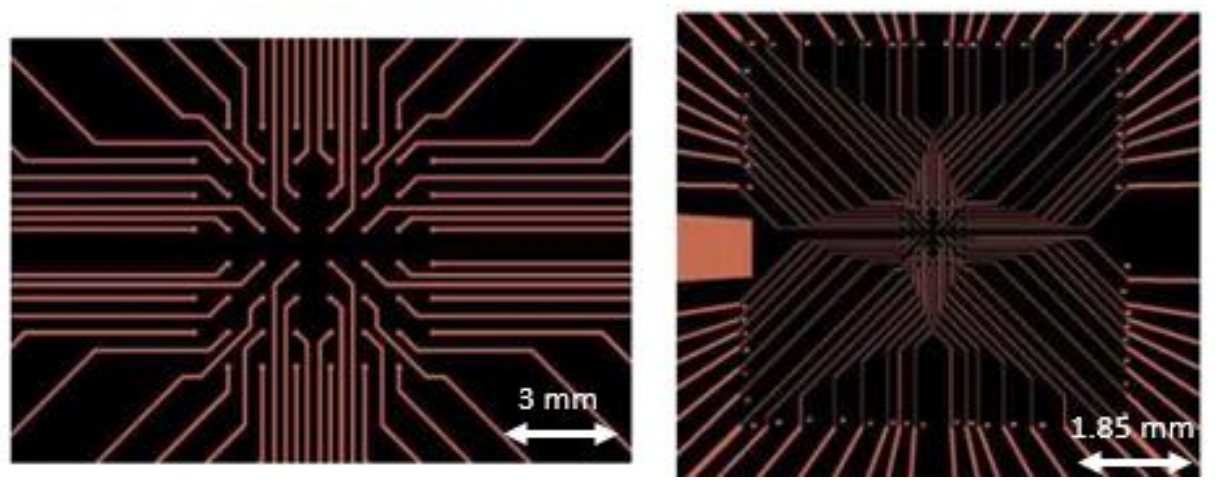

Figure 1. The pattern of electrodes in the proposed MEA that is designed in ADS software.

\section{Fabrication of Planar Multi-Electrode Array}

\subsection{Fabrication Consideration}

To manufacture the designed MEA, careful selection was made for the materials of substrate, conductor, insulator, and microelectrodes. In fact, in the fabrication process of multi-electrode arrays, several substantial criteria must be considered: First of all, all substances that come into contact with the cellular system must be biocompatible, optically transparent, rigid and durable to cell culture conditions. Secondly, since the amplitude of the action potential (AP) signals is relatively small, the parasitic impedance in the action potential signal sensing site and the signal path should be as low as possible. Thirdly, the insulation layer of the multi-electrode array must have a high impedance so that the action potential signal is not significantly attenuated. Finally, the measurement system should not have a negative effect on the physicochemical environment $[6,8]$.

When recording extracellular systems, it is best to keep the underside of the electrode transparent so that the system can be viewed under a microscope for further processes. In this work, we have used silica (glass) substrates as a very low-cost substrate in comparison to other substrates often chosen like Quartz $[9,10]$ or Si wafer $[11,12]$.

The material has a great effect on the electrical properties measured from the cells. Also, the adhesion of the material must be considered in the fabrication process of electrodes. In most related scientific articles, electrode selection is divided into two groups of metals and conductive polymers [5]. Polymers are generally used when a flexible electrode is needed to record living organisms. Such electrodes are usually more difficult to fabricate than metal electrodes. However, for extracellular recording, the use of metals is appropriate compared to polymers due to their strength and reusability. Common metals used in fabrication include Gold, platinum, and Titanium. These metals are biocompatible and resistant to corrosion [8,13-16].

After studying the adhesion, conductivity, and surface strength of the three available options of Gold, platinum, and Titanium, we used Gold because of its longevity. In other words, based on the durability and stability of the electrodes over time which has great importance, Gold was chosen in this study. The impedance of Platinum electrodes is low, but Gold is more stable than platinum and Titanium [15,17]. Additionally, Gold has other properties that make it suitable for coating medical instruments. These properties include high flexibility, non-oxidation, cell compatibility, high density, which makes it resistant to X-rays, high conductivity, and its reliable bonding to other metals.

However, because Gold does not adhere well to glass, a buffer layer such as nickel, Titanium or chromium should be used as an adhesion interface between Gold and glass $[4,8,14]$. In this work, we have chosen a thin layer of Titanium as the buffer layer metal.

Another important point in the fabrication of multi-electrode arrays is the use of efficient electrical insulators. Because the voltage leakage should be prevented as much as possible. Besides, 
the lack of insulation causes interference between the channels, which is problematic for recording. The insulation layer must have a low dielectric constant or a high thickness to reduce the parasitic capacitors formed between the cell culture medium and the electrode. Also, the insulating material must have an inert surface so that it does not react with the ions in the aqueous solution. [18].

In this work, we have chosen organic photoresist SU-8 as the insulator material. SU-8 has several applications in the deposition process such as the patterning of electrodes and their insulation. Also, due to its biocompatibility, it can be used directly as an insulating layer. This material has a relatively long life and its coating and etching methods are very simple to compare to other insulators like silicon nitride or dioxide materials [8].

Based on the fabrication considerations declared in this section and based on the materials chosen to manufacture the planar MEA electrode, the fabrication process is described in detail in the following section. All materials and processes are readily available and even cost-effective in caparison to other materials used in the microfabrication process.

\subsection{Fabrication Process}

Figure 2 shows a step-by-step process for the fabrication of the proposed Gold/Titanium multielectrode array chip. These steps include cleaning and preparing the sample surface, deposition of the buffer metal (Titanium), deposition of the Gold layer, adding the photoresist to the sample, and photolithography to create the desired design on the sample. Acid etching of metal layers in specific areas, adding an insulating layer to isolate the channels and insulating the connection paths and the surface are the next steps shown in Figure 2(7-11).

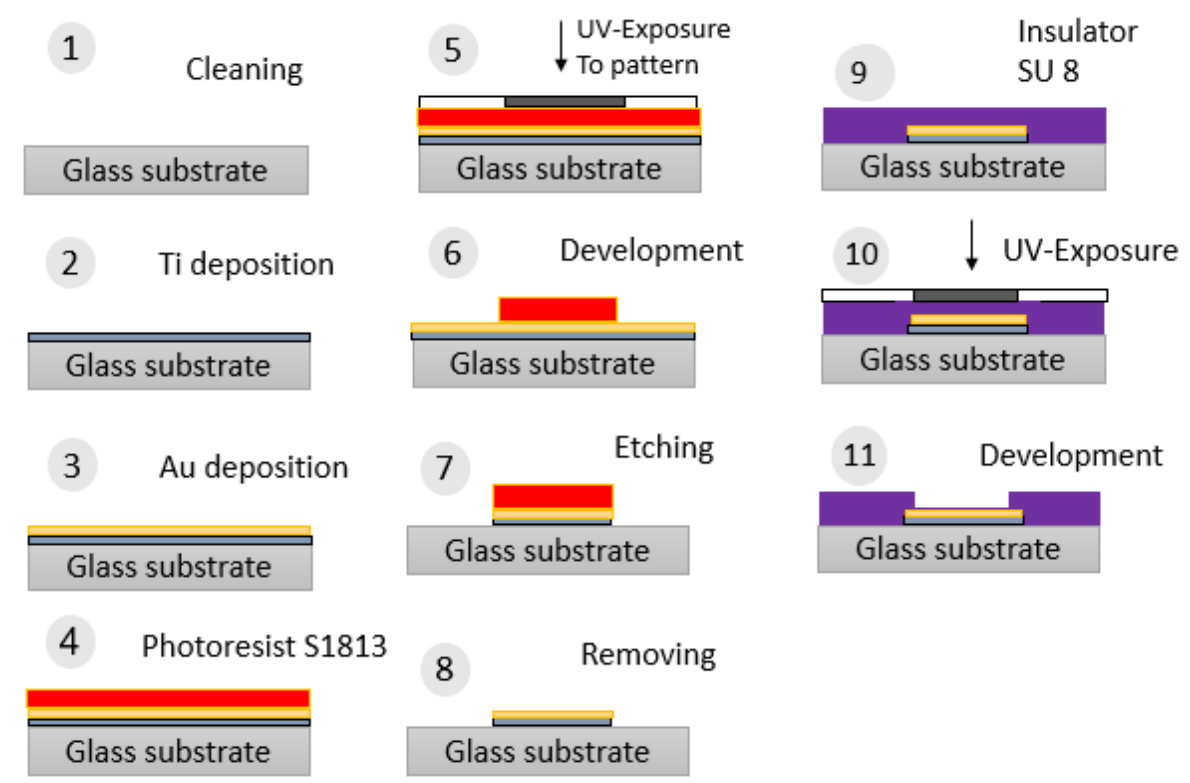

Figure 2. Proposed MEA fabrication step-by-step process.

Before the deposition of metal layers with the dc-sputtering coating technique, the substrate must be cleaned with acetone, high-purity isopropyl alcohol, and Piranha solution to remove a high percentage of contaminants. Since the substrate used in this study is glass, in order to degas it and improve the quality of the deposited layers, we must heat the sample in a high-temperature furnace before the sputtering process. Also, since the evaporation of water vapor from the surface of the substrate is an important factor in determining the geometric structure of the coating layers, the temperature of the substrate must be up to about $200^{\circ} \mathrm{C}$ during deposition [19].

The ratio of the thickness of Gold to the buffer Titanium layer should be such that it does not change the impedance and conductivity of the Gold electrodes and at the same time causes the proper adhesion of the Gold layer to the substrate. After sputtering of $120 \mathrm{~nm}$ Titanium in vacuum pressure 
of $2 \times 10^{-5}$ Torr, we can continue with the deposition of Gold layer. Afterwards, we deposit the $80 \mathrm{~nm}$ layer of Gold in pressure of $58 \mathrm{mTorr}$ on the Titanium coated glass substrate. This step was followed by a plasma treatment step using oxygen gas to activate the sample surface

After the standard photolithography process, using S1813 as the positive photoresist, and Aqua regia and dilute hydrofluoric acid as Gold and Titanium etchant respectively, the sample was ready for the deposition of the insulation layer. Except for the electrodes, the contact pads, the ground connection electrode, all other parts of the chip should be insulated. A thin biocompatible insulation layer (Microchem SU-8 photoresist) was deposited on the sample. By heating it to high temperature, it becomes very rigid and can be used in signal recording for several months.

The final step is locating the glass ring as a well for the placement of the cell on the sample, which adhesion should be resistant to heat and laboratory operations. After adhering the ring to the substrate, the outer wall of the ring was covered by a thin layer of SU-8 protective insulation material to ensure the insulation and biocompatibility of the sample surface.

Figures 3-5 show a final multi-electrode array chip, and microscopic view of electrodes and connection lines structures, respectively.

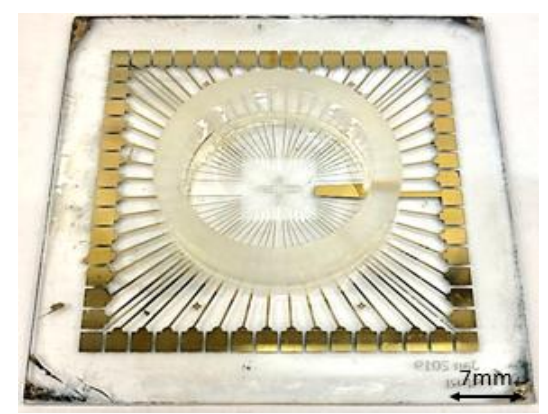

Figure 3. Final structure of multi-electrode array chip.
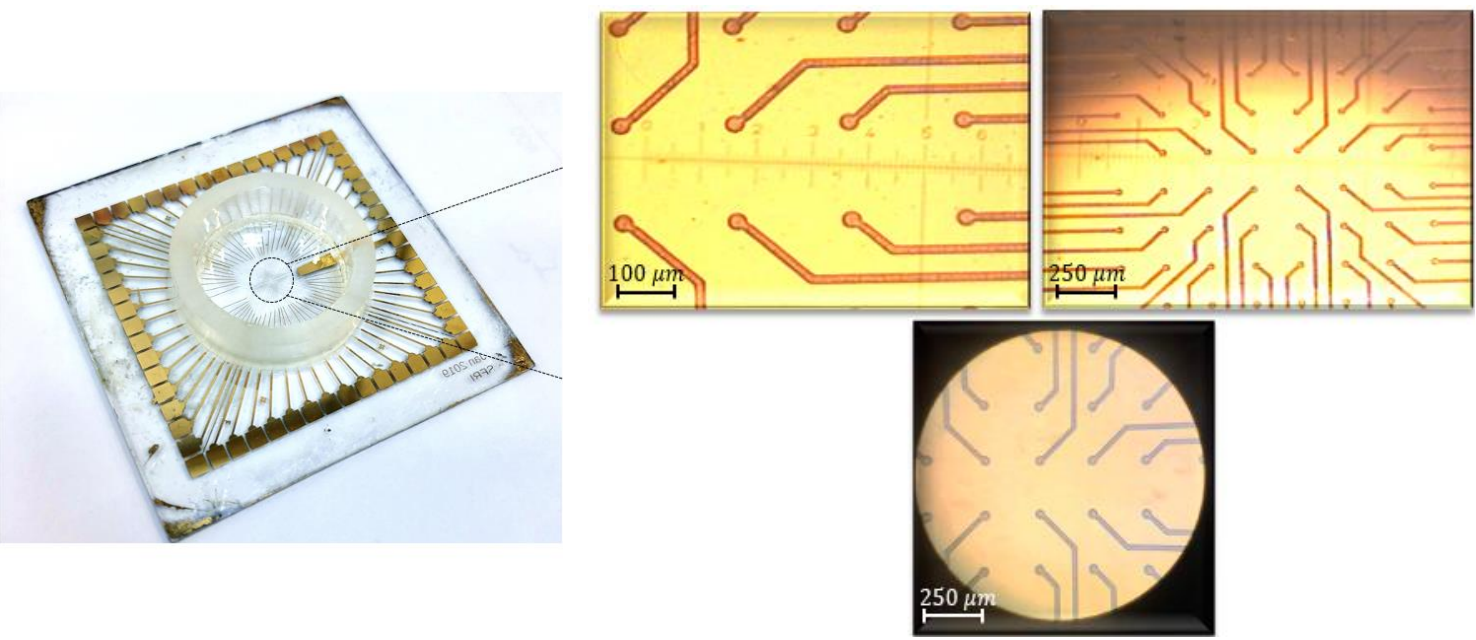

Figure 4. Microscopic view of the fabricated electrodes.
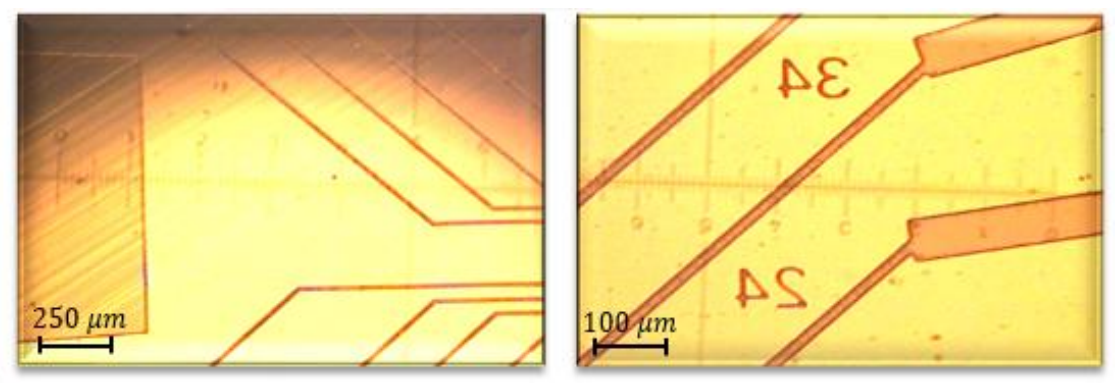

Figure 5. Microscopic view of the connection lines. 


\section{MEA Preparation Prior to Neural Cell Culture}

The manufactured MEA was washed via Terg-a-zyme ${ }^{\circledR}$ detergent (Z273287; Sigma-Aldrich) on a vertical shaker for $1 \mathrm{~h}$ at room temperature. Afterwards, the chip was sterilized inside $70 \%$ ethanol for $15 \mathrm{~min}$ and then left to dry under laminar flow cabinet. For the perfect attachment to be accomplished between neurons and glass, the MEA surface ought to be treated with appropriate proteins prior to cell seeding. Therefore, sterile MEA was coated by Poly-L-ornithine (PLO, P4957; Sigma) plus Laminin (L2020, Sigma) in $37^{\circ} \mathrm{C}$ for one and three $\mathrm{h}$ respectively.

\subsection{Neural Cell Isolation from Rat Cortices}

Cultured neurons on MEA were isolated from rat cerebral cortex between E16-18 gestational stage. The isolation process was executed according to the previously described protocol with several modifications [20]. In brief, a pregnant rat was euthanized in compliance with institutional guidelines. In the next step, the animal's abdominal skin and peritoneum were cut respectively to expose the abdominal cavity. Then, the embryo containing uterus was transferred into ice-cold dissection buffer comprising of: Hank's Balanced Salt Solution (HBSS, 14025-050; Gibco) + $20 \mathrm{mM}$ Glucose (G7021; Sigma) + 1\% GlutaMAX (35050-038; Gibco) + 100 U/mL Penicillin-Streptomycin + 50 $\mathrm{mg} / \mathrm{mL}$ Gentamycin (15750-060; Gibco). All embryos were decapitated immediately via sharp scissors. Thereafter, in order to expose the cortical lobes, the head skin as well as the skull tissue were removed using a micro scissors. Eventually, the superficial layer of the cortices was dissociated from the whole brain via small short curved forceps and was immersed inside the Phosphate-buffer saline (PBS-, 10010-031; Gibco) supplemented with: 20 mM Glucose (G7021; Sigma) + 1\% GlutaMAX (35050038; Gibco) + $100 \mathrm{U} / \mathrm{mL}$ Penicillin-Streptomycin + $50 \mathrm{mg} / \mathrm{mL}$ Gentamycin (15750-060; Gibco). In the following, the covering meninge was removed accurately to prevent blood cell contamination and then the cortical tissue was dissected into small pieces. In the next step, neuronal cells were isolated from the small tissue pieces by enzymatic treatment, consuming $0.25 \%$ Trypsin-EDTA (15400-054; Gibco) for $20 \mathrm{~min}$ at $37^{\circ} \mathrm{C}$.

\subsection{Neural cell Culture on MEA}

The isolated neurons were nourished by NeurobasalTM Medium (21103-049; Gibco) supplemented with 2\% B27 (17504-044; Gibco) + 1\% horse serum (H1138; Sigma) + 1\% GlutaMAX (35050-038; Gibco) + $100 \mathrm{U} / \mathrm{mL}$ Penicillin-Streptomycin + $20 \mathrm{mM}$ Glucose (G7021; Sigma). The medium was exchanged completely after $24 \mathrm{~h}$ of cell seeding and then in a half-half manner every other day.

\section{Results}

The MEA preserved a robust structure during pre-culture treatments. The preliminary evaluation of the manufactured MEA were performed so as all the features were compared to the original standard MEA chip purchased from Multichannel system company. The MEA was washed perfectly in order to remove cell debris as well as contaminates from the surface. Also, it was sterilized to prevent further cell contamination before cell culture. During the washing and sterilizing process, we did not observe detachment of the ring from the surface or any fracture on the whole product. Moreover, the arrays of the electrode seemed to retain intact after the washing and sterilization under the microscope (Figure 6). The higher temperature of the incubator $\left(37^{\circ} \mathrm{C}\right)$ did not affect the ring adhesion robustness as well, during the coating process. 

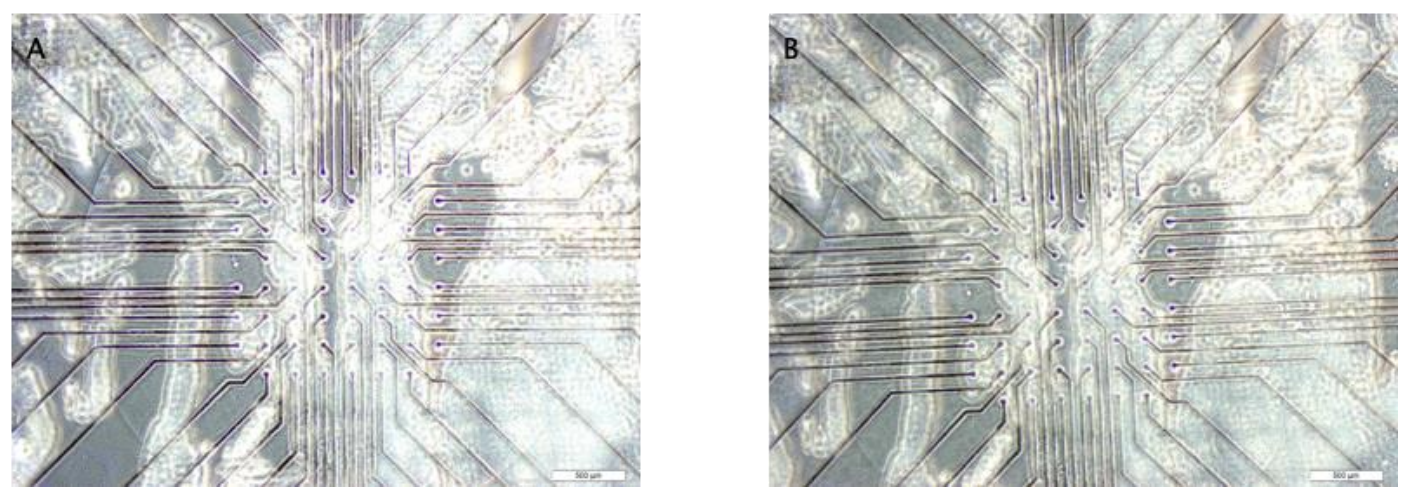

Figure 6. The microscopy studies revealed the preserved microstructure of the array before and after washing and sterilization in manufactured MEA.

The biocompatibility of the MEA is going to be addressed. To evaluate the biocompatibility of the MEA, we isolated neural cells from rat cortices. After neural isolation, around $400 \times 10^{3}$ cells, suspended in $1 \mathrm{ml}$ medium, were seeded on the MEA. Cell attachment to the surface will be assessed visually via light microscopy and the percentage of the cell viability will be determined by MTS assay in the next step of the study.

\section{Conclusions}

In this paper, we have designed and manufactured a low-cost planar Gold/Titanium multielectrode array chip on a silica substrate with 60 electrodes of $30 \mathrm{~mm}$ diameter and the biocompatible insulation layer of SU-8 using standard microfabrication methods. The choice of substrate, method, and materials used is based on a cost-effective product. In other words, glass because of its low cost, gold because of its stability and high biocompatibility, and SU-8 because of the simplicity of the manufacturing process as well as its biocompatibility were chosen. The multi-electrode array was prepared using these materials and the standard non-expensive microfabrication method. In this study, the biocompatibility of the manufactured MEA is to be assessed for long time by neural primary culture. The aim is to monitor the cell's attachment to the glass surface as well as the ability of the neural cells to freely extend their dendrite and axons in a natural timeline, via light microscopy. Moreover, the accurate percentage of cell death will be stochasticaly assessed for long times by MTS assay in the next step to illustrate the influence of the consuming materials on neural cell survival.

Funding: This research was partially supported by the Iranian National Science Foundation (INSF).

\section{References}

1. Pas, J. Flexible neural probes with a fast bioresorbable shuttle from in vitro to in vivo electrophysiological recordings. 2017.

2. Regalia, G.; Biffi, E.; Ferrigno, G.; Pedrocchi, A. A Low-Noise, Modular, and Versatile Analog Front-End Intended for Processing In Vitro Neuronal Signals Detected by Microelectrode Arrays. Comput. Intell. Neurosci. 2015, 2015, 1-15.

3. Chang, Y.J.; Liao, L.D.; Lin, C.T.; Lai, H.Y.; Chen, J.L.; Yang, Y.T.; Ting, Y.C.; Huang, Y.P.; Wu, R.; Thakor, N.V.; et al. A Low-Cost Multi-Electrode Array System for the Simultaneous Acquisition of Electrophysiological Signal and Cellular Morphology. J. Neurosci. Neuroeng. 2012, 1, 131-142.

4. Kang, H.; Nam, Y. Stretchable Bioelectronics for Medical Devices and Systems; Springer: Cham, Switzerland, 2016; pp. 275-291.

5. Müller, J.; Ballini, M.; Livi, P. High-resolution CMOS MEA platform to study neurons at subcellular, cellular, and network levels. Lab Chip 2015, 15, 2767-2780.

6. Kim, R.; Joo, S.; Jung, H.; Hong, N.; Nam, Y. Recent trends in microelectrode array technology for in vitro neural interface platform. Biomed. Eng. Lett. 2014, 4, 129-141.

7. Multi Channel Systems MCS GmbH. Microelectrode Array (MEA) Manual. Microelectrode Array (MEA) Manual; 2017; p. 68. 
8. Zhang, X.; Zhang, Y.; Li, Y.; Nelson, R.D.; LaRue, J.C. Development of planar microelectrode array for extracellular characterization of action potentials in sinoatrial node cells. In Proceedings of the 2011 International Symposium on Bioelectronics and Bioinformatics, ISBB 2011, 2011; pp. 1-4.

9. Xie, C.; Lin, Z.; Hanson, L.; Cui, Y.; Cui, B. Intracellular recording of action potentials by nanopillar electroporation. Nat. Nanotechnol. 2012, 7, 185-190.

10. Lin, Z.C.; Xie, C.; Osakada, Y.; Cui, Y.; Cui, B. Iridium oxide nanotube electrodes for sensitive and prolonged intracellular measurement of action potentials. Nat. Commun. 2014, 5, 3206.

11. Gabay, T.; Ben-David, M.; Kalifa, I.; Sorkin, R.; Abrams, Z.R.; Ben-Jacob, E.; Hanein, Y. Electro-chemical and biological properties of carbon nanotube based multi-electrode arrays. Nanotechnology 2007, 18, 035201.

12. Robinson, J.T.; Jorgolli, M.; Shalek, A.K.; Yoon, M.H.; Gertner, R.S.; Park, H. Vertical nanowire electrode arrays as a scalable platform for intracellular interfacing to neuronal circuits. Nat. Nanotechnol. 2012, 7, 180184.

13. Kim, R.; Hong, N.; Nam, Y. Gold nanograin microelectrodes for neuroelectronic interfaces. Biotechnol. J. 2013, 8, 206-214.

14. Obien, M.E.J.; Deligkaris, K.; Bullmann, T.; Bakkum, D.J.; Frey, U. Revealing neuronal function through microelectrode array recordings. Front. Neurosci. 2015, 9, 423.

15. Seker, E.; Berdichevsky, Y.; Begley, M.R.; Reed, M.L.; Staley, K.J.; Yarmush, M.L. The fabrication of lowimpedance nanoporous gold multiple-electrode arrays for neural electrophysiology studies. Nanotechnology 2010, 21, 125504.

16. Kim, R.; Joo, S.; Jung, H.; Hong, N.; Nam, Y. Recent trends in microelectrode array technology for in vitro neural interface platform. Biomed. Eng. Lett. 2014, 4, 129-141.

17. Berdondini, L.; Chiappalone, M.; Van Der Wal, P.D.; Imfeld, K.; de Rooij, N.F.; Koudelka-Hep, M.; Tedesco, M.; Martinoia, S.; Van Pelt, J.; Le Masson, G.; et al. A microelectrode array (MEA) integrated with clustering structures for investigating in vitro neurodynamics in confined interconnected sub-populations of neurons. Sens. Actuators B: Chem. 2006, 114, 530-541.

18. Kang, H.; Nam, Y. Stretchable Bioelectronics for Medical Devices and Systems; 2016; pp. 275-291.

19. Riester, M.; Barwulf, S.; Lugscheider, E.; Hilgers, H. Morphology of sputtered titanium nitride thin films on thermoplastic polymers. Surf. Coat. Technol. 1999, 116, 1172-1178.

20. Kim, H.J.; Magrané, J. Isolation and Culture of Neurons and Astrocytes from the Mouse Brain Cortex. In Neurodegeneration; Humana Press: Totowa, NJ, USA, 2011; pp. 63-75.

Publisher's Note: MDPI stays neutral with regard to jurisdictional claims in published maps and institutional affiliations.

(C) 2020 by the authors. Submitted for possible open access publication under the terms and conditions of the Creative Commons Attribution (CC BY) license (http://creativecommons.org/licenses/by/4.0/). 